\title{
Concatenated RS-Convolutional Codes for Cooperative Wireless Communication
}

\author{
Yahya Mohammed Hameed Al-Moliki ${ }^{1, *}$, Kamarul Ariffin Bin Noordin ${ }^{1}$, MHD Nour Hindia ${ }^{1}$ and \\ Mohd Fadzli Bin Mohd Salleh ${ }^{2}$
}

\author{
${ }^{I}$ Department of Electrical Engineering, University of Malaya, 50603 Kuala Lumpur, Malaysia \\ ${ }^{2}$ School of Electrical and Electronic Engineering, Universiti Sains Malaysia, 14300, Pinang, Malaysia
}

\begin{abstract}
In the last few years, several works have investigated the performance of cooperative wireless systems with various types of channel codes such as Low Density Parity Check (LDPC), Turbo, CRC, convolutional and recently Reed Solomon (RS) codes. These cooperative schemes give power to single antenna mobiles to originate virtual multiple antennas transmitter, MIMO system, by sharing their antennas so that diversity gain is achieved. The most common cooperative schemes are decode and forward (DF) and coded cooperation (CC). Concatenated Reed Solomon and convolutional codes have been used by several wireless communication standards like digital video broadcasting (DVB) standards and IEEE 802.16e WiMAX standard. In this paper, we develop two different coded cooperation schemes and apply them to the mandatory structure of physical layer specifications of IEEE 802.16e WiMAX. The results attained for both coded cooperation schemes present magnificent diversity gains over non-cooperative scheme.
\end{abstract}

Keywords: WiMAX, IEEE 802.16e, Reed Solomon codes, Convolutional codes, Interleaver, Cooperative, Coded Cooperation, Rayleigh Fading.

\section{INTRODUCTION}

\subsection{Background}

In wireless communication channels, mobile signals are affected by fading. It means the power of the signal is attenuated and fluctuated significantly according to channel conditions due to fading. Thus, the performance of the wireless communication systems is degraded. Diversity can overcome the effect of fading and can be generated by sending out independent copies of the signals from different locations, spatial diversity.

To achieve transmit diversity more than one antenna is required at the transmitter. However, many mobile devices cannot support multiple antennas due to size, cost and complexity. So another manner, cooperative communication, is used to perform transmit diversity by enabling single antenna mobiles to cooperate by sharing their antennas to create virtual multiple antennas transmitter, MIMO system, in the uplink of a wireless system as shown in Fig. (1).

Cooperative communication improves the quality of service that is evaluated at the physical layer such as bit error rates, block error rates or outage probability.

The fundamental signaling techniques for cooperative communication are amplify and forward (AF), decode and forward (DF) and coded cooperation (CC).

Amplify and forward (AF) is the simplest method of cooperation owing to its low intricacy. In this method, each

*Address correspondence to this author at the Department of Electrical Engineering, University of Malaya, 50603 Kuala Lumpur, Malaysia; Tel: 00601112338503; E-mail: almoliky_yahya@hotmail.com user receives a noisy version of the signal transmitted by its partner and then amplifies and resends this noisy signal to the base station. At the base station, both noisy signals transmitted by the user and its partner are combined to implement maximum ratio combining so that a final judgment is resolved on the user's transmitted bits. This technique was first designed by Laneman et al. [1].

Decode and forward (DF) is a cooperative signaling technique in which a user tries to decode the partner's data and then resend the decoded data to the base station. A. Sendonaris et al. $[2,3]$ proposed a simple code-division multiple access application of decode and forward coded cooperative communication system. The IEEE 802.16e WiMAX standard adopts DF system for cooperative communication.

The previous methods, AF and DF, enhance the capacity and outage probability for cooperating nodes. However, the repetition of the source's transmission is included during cooperation, which may not be the optimum utilize of the available channel. Also they may forward erroneous estimates of the partner's symbols or comprise transmission of the partner's noise. As a result, the performance is reduced due to error propagation especially when the inter-user channel begins to be poor. Furthermore, they involve the base station to know either the instantaneous (BER) or signal to noise ratio (SNR) of the inter-user channel to achieve optimal maximum likelihood (ML) detection or decoding [4].

The limitations due to previous schemes can be avoided by using cooperative signaling that takes place as a part of channel coding, coded cooperation (CC).

Coded cooperation (CC) is the latest signaling techniques for cooperative communication. It is carried out through 


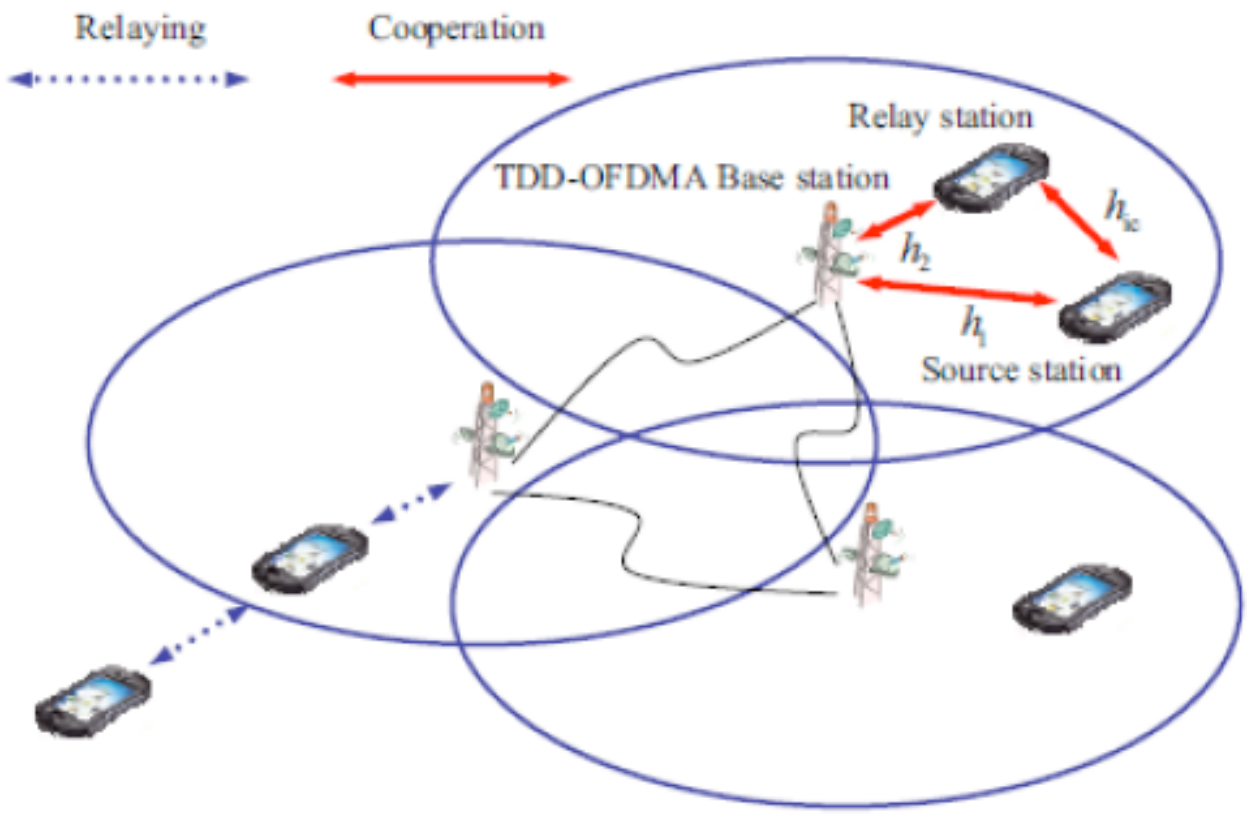

Fig. (1). Cooperative communication in wireless networks.

Amplify and forward Decode and forward

Coded cooperation
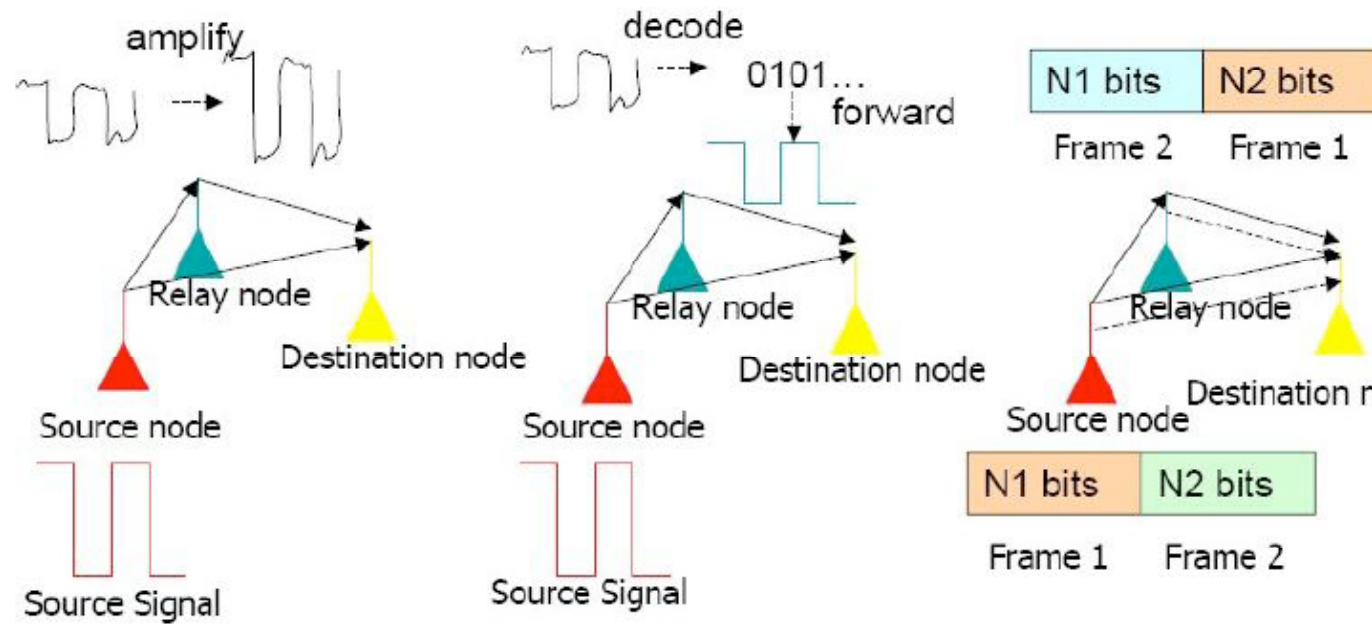

Source node

Destination node

N1 bits N2 bits

Frame 1 Frame 2

Fig. (2). Cooperative diversity schemes.

channel coding rather than a direct relay or repetition. Each code-word $\mathrm{N}$ is partitioned into two segments, N1 and N2. $N 1$ is transmitted by the user itself and $N 2$, the puncture bits, is transmitted by its partner. (CC) accomplishes magnificent gain relative to a non-cooperative system with the same information rate, transmit power and bandwidth. In coded cooperation, the standard channel coding techniques are utilized to perform cooperation in order to improve diversity. Todd E. Hunter et al. [5-7] investigated a coded cooperation system that uses the Rate-Compatible Punctured Convolutional codes (RCPC).

Fig. (2) shows the operation of cooperative diversity techniques.

Concatenated RS- convolutional codes (RSCC) have been adopted by various wireless communication standards.
FEC with Reed Solomon coding and convolutional coding is a powerful method to reduce the noise [8].

Due to the limitations, mentioned above, of DF systems utilized in some wireless communication standards such as IEEE 802.16e WiMAX standard, this work proposes two coded cooperation (CC) systems applied to the mandatory features of the IEEE 802.16e WiMAX specifications, channel coding with Reed Solomon and convolutional coders (RSCC), to attain less bit error rate (BER) and more diversity gains over non-cooperative scheme.

\subsection{WiMAX Overview}

The mandatory channel coding structure of the specification of IEEE 802.16e and ETSI HiperMAN consists of three parts randomization, Forward Error Correction (FEC) and interleaving. 


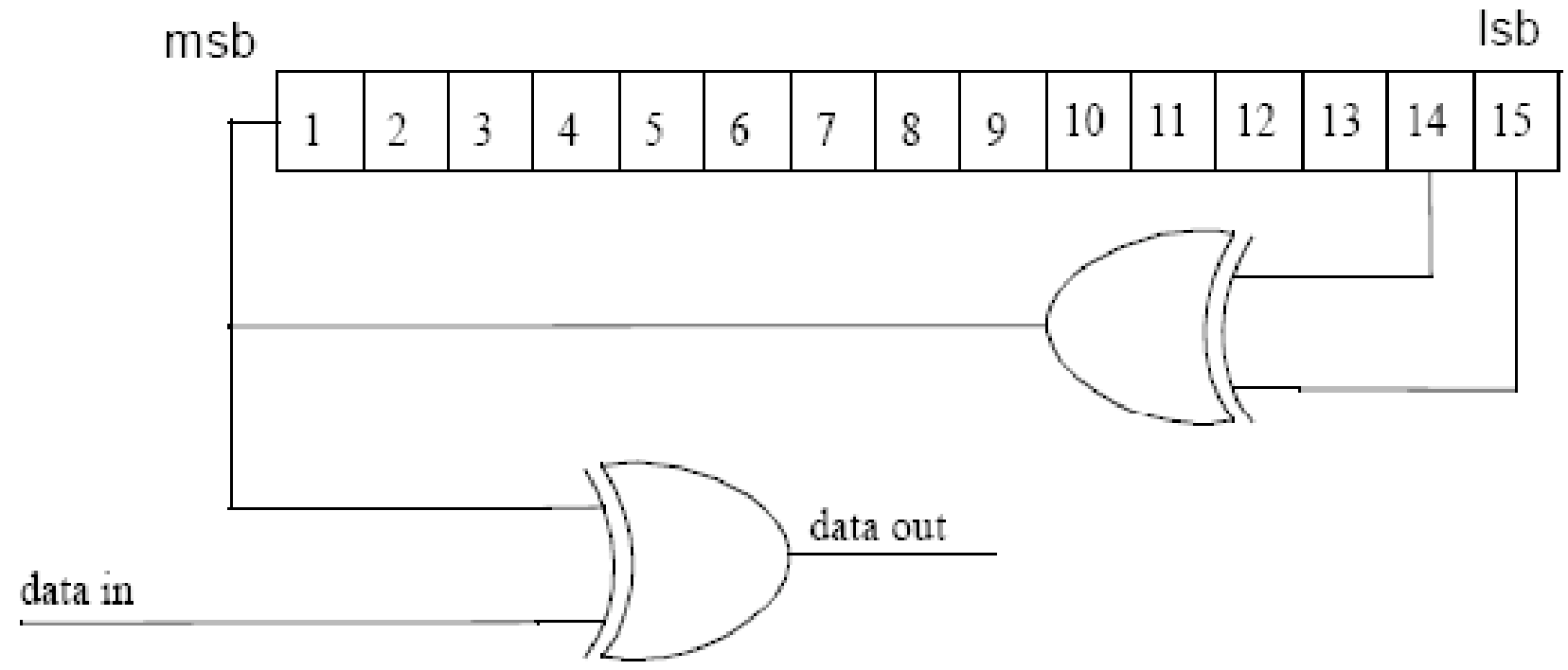

Fig. (3). Randomizer.

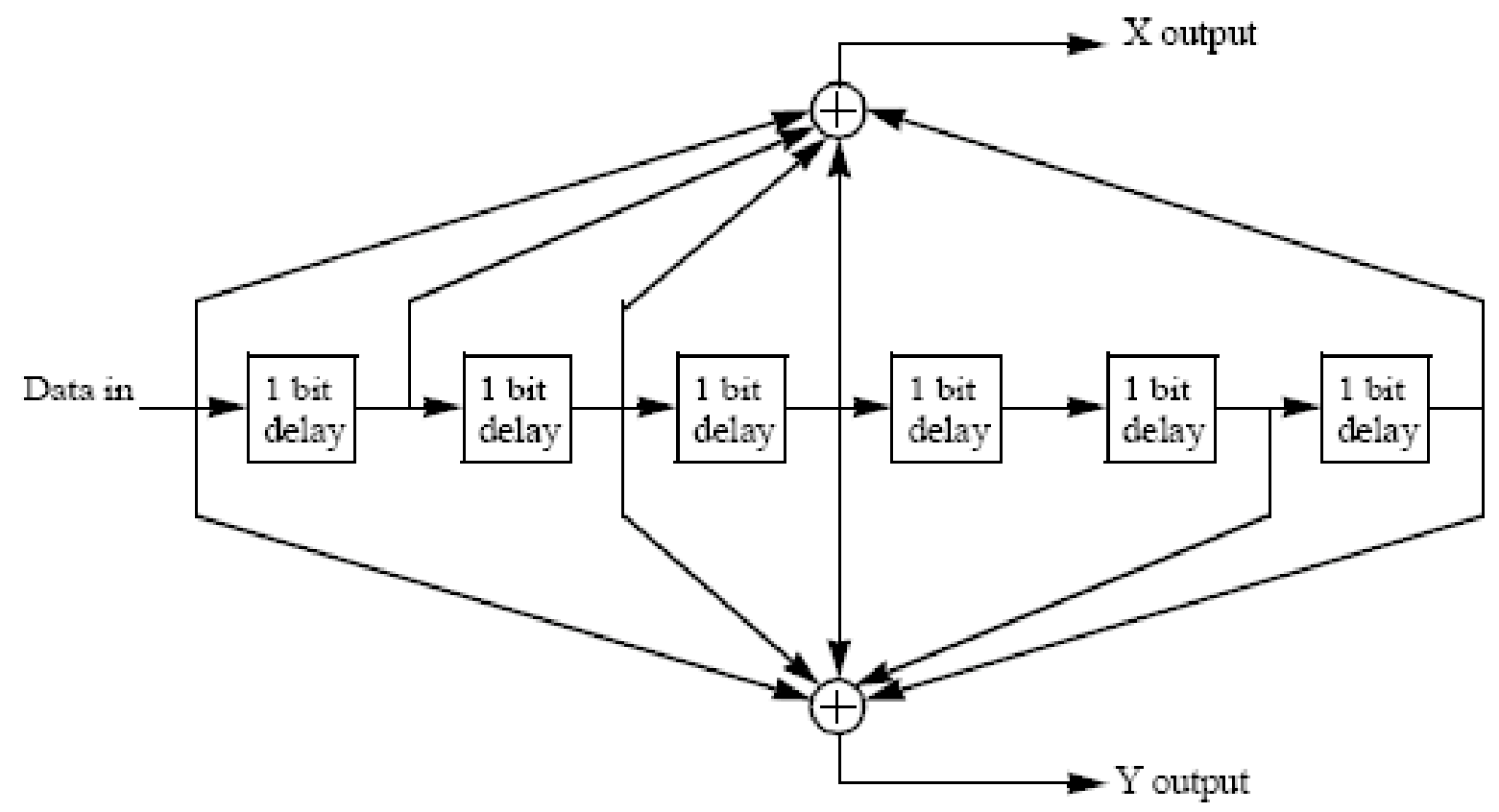

Fig. (4). Convolutional Encoder.

\subsubsection{Randomization}

Randomizer is used to avoid long sequences of consecutive ones or zeros via randomization of each data burst on each allocation. It can be carried out with a Pseudo Random Binary sequence generator. It consists of 15-stage shift register with generator polynomial of $1+x^{14}+x^{15}$ and initial state of

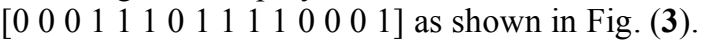

\subsubsection{Reed Solomon Coding}

The randomized data is passed in block format to RS encoder after appending a single 0X00 tail byte to the end of each burst. A systematic RS $(N=255, K=239, T=8)$ with GF $\left(2^{8}\right)$ is implemented in WiMAX, which it can correct up to eight symbol errors per block. Two polynomials, primitive and generator polynomials, are employed for code generator and field generator. Primitive polynomial is $1+x^{4}+x^{5}+x^{6}+x^{8}$ and generator polynomial is. $1+x^{2}+x^{3}+x^{4}+x^{8}$.
The encoder can generate shortened and punctured code to support variable block sizes and variable error correction capability. Before the data block, 239-K zero bytes are added to generate a shortened code and after encoding, these zero bytes are removed. The punctured code is then generated for which specific parity symbols are discarded from the output of the encoder.

\subsubsection{Convolutional Encoder}

The RS output code-word is fed to inner binary convolutional encoder that has $1 / 2$ coding rate and 7 constraint length as shown in Fig. (4). The generator polynomial is determined by $\mathrm{G} 1=171$ OCT for $\mathrm{X}$ and $\mathrm{G} 2=133$ OCT for $\mathrm{Y}$.

By puncturing operation, different code rate can be obtained from the output of convolutional encoder. In the standard, three different code rate can be achieved 2/3, 3/4 and $5 / 6$. 


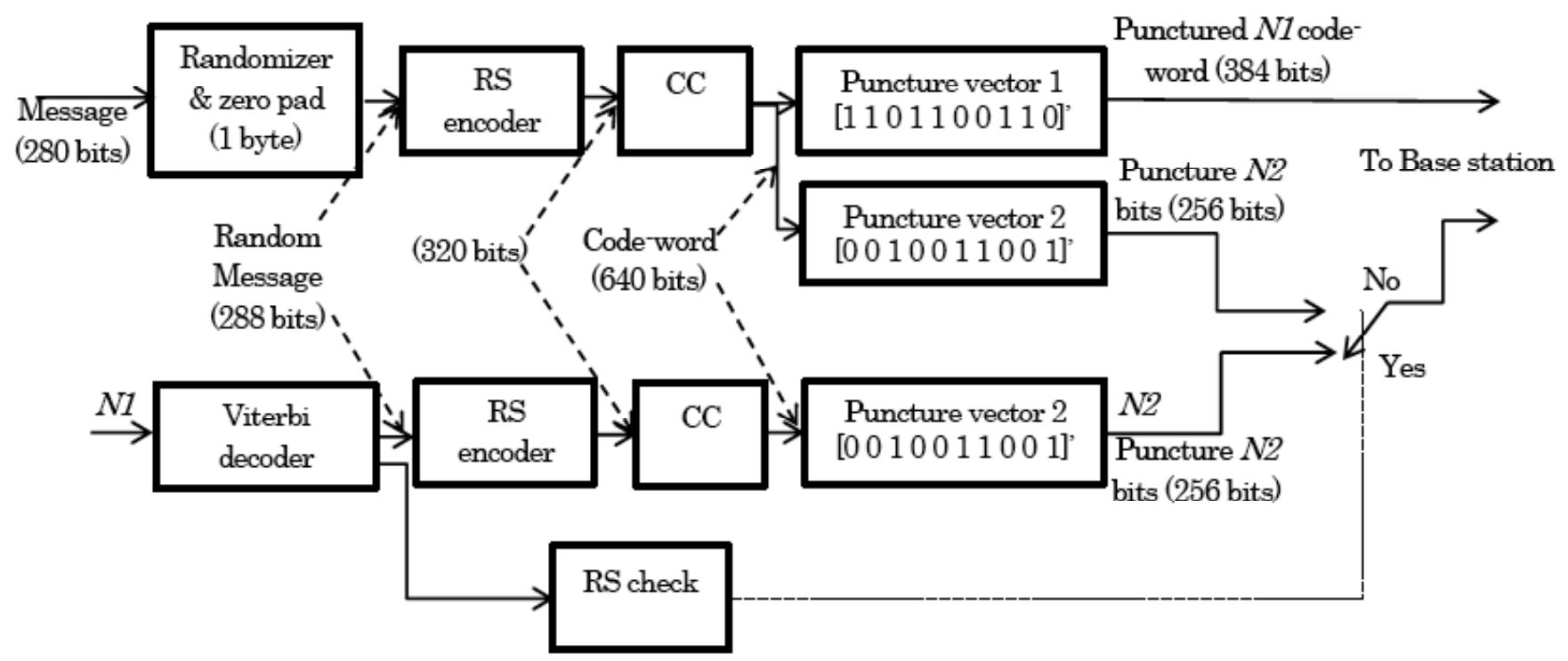

Fig. (5). Coded cooperation at Source and Relay, code rate $R=0.45$ (model 1).

\subsubsection{Interleaver}

The output of RSCC encoder is interleaved by a block interleaver. The block size corresponds to the length of the coded data, RSCC output, per allocated sub-channel per OFDM symbol. The interleaver in IEEE $802.16 \mathrm{e}$ is made of two steps permutation.

- Permute the coded bits where the adjacent bits are mapped onto nonadjacent subcarriers.

- Permute the adjacent coded bits where they are mapped alternatively onto less or more significant bits of the constellation to avoid long runs of unreliable bits.

The index value of the bits after two step permutation can be calculated by the Matlab implementation using the following equations.

$\mathrm{fk}=(\mathrm{Ncbps} / 12) * \bmod (\mathrm{k}, 12)+$ floor $(\mathrm{k} / 12)$

$\mathrm{s}=\operatorname{ceil}(\mathrm{Ncpc} / 2)$

$\mathrm{sk}=\mathrm{s} *$ floor $(\mathrm{fk} / \mathrm{s})+\bmod (\mathrm{s}, \mathrm{fk}+\mathrm{Ncbps}-\operatorname{floor}(12 *$ $\mathrm{fk} / \mathrm{Ncbps})$ )

[s,int_idx] $=$ sort (sk)

In this paper, we propose two coded cooperation models that are designed on the basis of the mandatory channel coding of IEEE 802.16e WiMAX, RS codes concatenated with convolutional codes.

\section{THE FIRST MODEL}

Unlike the work demonstrated in [7] and [8], where Hunter uses cyclic redundancy check (CRC) and convolutional codes to perform a coded cooperation method, we propose the same cooperation mechanism with different codes, RSCC, as well as we follow the IEEE 802.16e WiMAX standard.

At the source, $N$ bits code-word is punctured to $N 1$ bits code-word that is broadcasted to the relay and the base station through direct and inter-user channels at the first time step. The relay tries to recover the source's message from the received $\mathrm{Nl}$ code-word. If the relay successfully decodes the source data, it re-computes the other partition of $N 2$ bits which is transmitted through the relay channel to the base station at the second time step. N1 and N2 segments reach the base station and then they are combined to form the $N$ code-word that is decoded to recover the source message. In other words, if the relay cannot correctly decode the source data, the system reverts to non-cooperative operation. At this state, the second partition of $N 2$ bits is transmitted by the source itself to the base station at the second time step. This model provides diversity gain while maintaining the same information rate and bandwidth. Fig. (5) shows the main components of this system that uses the mandatory specification of IEEE 802.16e WiMAX channel codes, RSCC. The coded cooperation at each node is discussed as follows.

\subsection{Source}

The output of convolutional encoder, $N$ code-word, is punctured to Nl code-word. The puncturing can be performed by the vector arithmetic operation. The puncture vector that is used at this design is $\left[\begin{array}{llllllllll}1 & 1 & 0 & 1 & 1 & 0 & 0 & 1 & 1 & 0\end{array}\right]$ ', called puncture vector 1 . This vector reshapes ( $N$ code-word) to produce the first partition Nl code-word, the first frame. By this vector, the third, sixth, seventh and tenth elements are removed from the group of ten input elements of $N$ codeword vector while the others are conserved. The first frame is broadcasted through Rayleigh fading channels to the relay and base station.

\subsection{Relay}

If the relay decodes the source's data successfully, it starts to re-compute the other partition of $N 2$ bits. N2, puncture bits, can be produced by reshaping the $\mathrm{N}$ code-word by

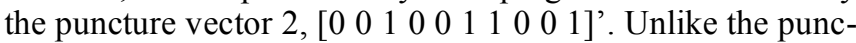
ture vector 1 at the source, each one in the puncture vector 1 is replaced by zero and each zero is replaced by one. By puncture vector 2, the other partition N2 segment is obtained. Then this puncture bits $N 2$, the second frame, are sent to base station through Rayleigh fading (relay) channel. 

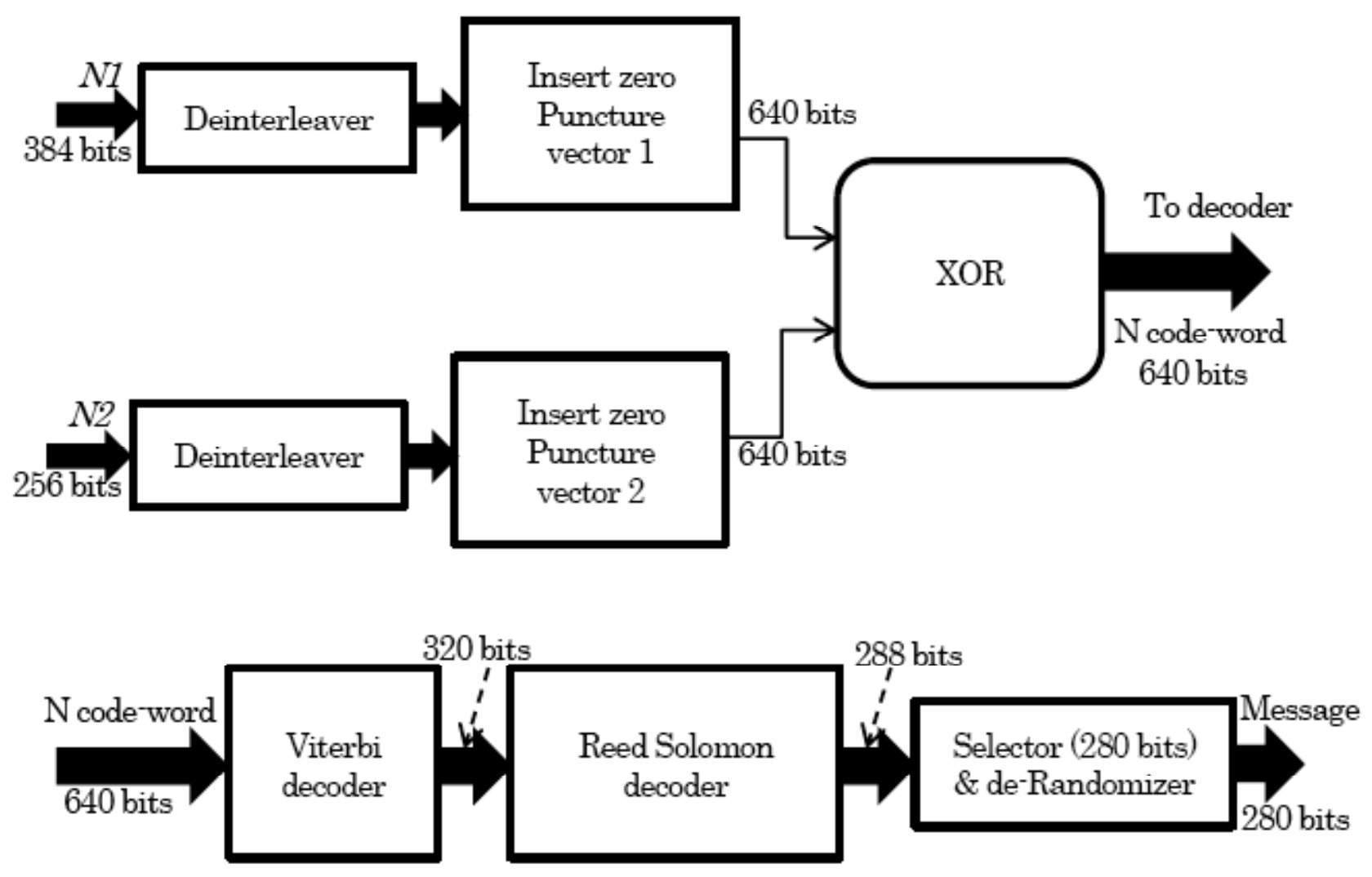

Fig. (6). Combination and decoding operations at Base station, code rate $R=0.45$ (model 1).

As shown in Fig. (5), RS checks if the relay node decodes the source data successfully. When the operation of decoding becomes incorrect due to the condition of the interuser channel or other reasons, the system returns to noncooperative mode. The puncturing rate can be adapted according to the condition of the inter-user channel so that the relay can successfully decode the source's transmission.

\subsection{Base station}

Both partitions are combined at the base station to generate the original $N$ code-word. The operation of combination is demonstrated in Fig. (6).

In Fig. (6), zero puncture vector $\left.1\left(\begin{array}{llllllllll}1 & 1 & 0 & 1 & 1 & 0 & 0 & 1 & 1 & 0\end{array}\right]\right)$ inserts zeros among the elements of the N1 code-word vector, where each zero in this vector means that zero should be added at that location of each sequential grouping of six input elements of $N 1$ code-word. On the other hand, each one implies that the coming element of the input N1 code-word vector should be placed at the output of "insert zero block". Also zeros are inserted in the second partition, $N 2$ bits, by

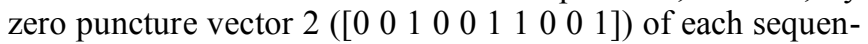
tial grouping of four input elements of $N 2$ bits. Then the resultant vectors are added by XOR operation to create the original $N$ code-word.

Fig. (6) shows also the operation of decoding that is implemented at the base station. $N$ code-word is applied to Viterbi and Reed Solomon decoders to recover the source's message in the output of De-randomizer. For Viterbi decoders in the relay and the base station to work well, eight zero bits are appended to the end of each burst at the source. This single tail byte is required for decoding algorithm operation.

\section{THE SECOND MODEL}

In the second design, the original $N$ code-word is partitioned into two segments $N 1$ and $N 2$ bits at the source. The first partition, N1 code-word, is sent directly to the base station at the first time step while the second partition, N2 puncture bits, is encoded again by Reed Solomon and Convolutional encoders (RSCC1) at the source and then is sent to the relay. At the relay, the encoded $N 2$ segment is decoded by Viterbi and RS decoders to recover N2, puncture bits, punctured at the source. If the decoding is successful, decided by RS decoder, both relay and source nodes send the encoded N2 segments to the base station at the second time step. If the decoding is not successful, the relay node stays inactive. At the base station, MRC or selection diversity is implemented on the received encoded N2 segments [9]. Then the output of MRC is de-interleaved and decoded to recover the source's message. The coded cooperation scheme at each node is discussed as follows.

\subsection{Source}

Fig. (7) illustrates the operation of coded cooperation utilized at the source. Message is randomized and encoded by RSCC1 to generate $N$ code-word. Then $N$ code-word is interleaved by interleaver 1 . Interleaver permutes encoded bits according to a mapping to avoid symbol error burst. After interleaving, $N$ code-word is separated into two segments, $N 1$ and N2. N1 segment is directly transmitted through Rayleigh fading (direct) channel to the base station while N2 segment is encoded again after appending a single 0X00 tail byte required for Viterbi decoding algorithm at the relay and the base station. Then $N 2$ is transmitted after interleaving by 


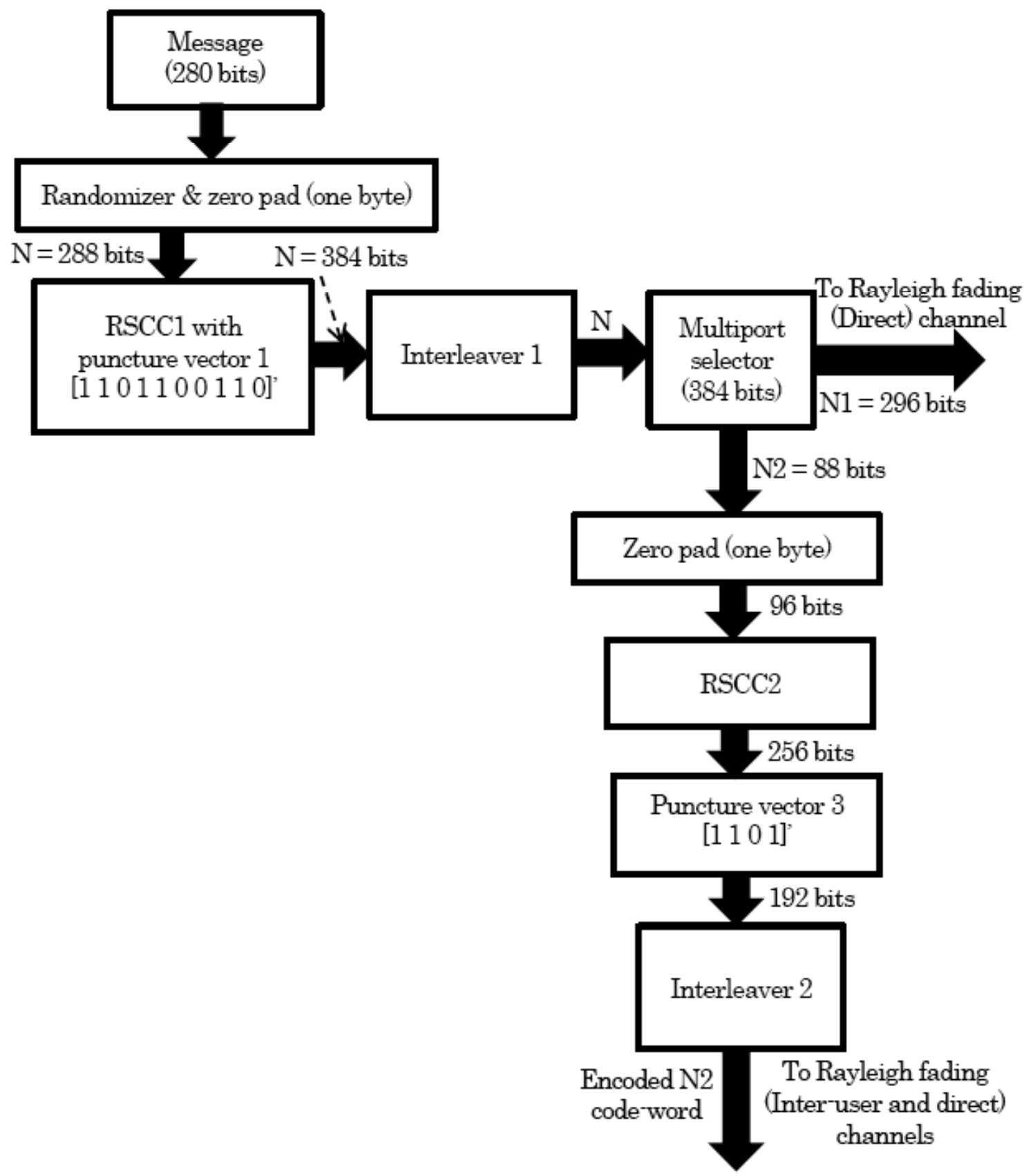

Fig. (7). Coded cooperation at Source, (88/384) cooperation level, code rate $R \approx 3 / 5$ (model 2).

interleaver 2 through Rayleigh fading (Inter-user) channel to the relay node. $N 2$ segment that is sent to the relay (partner) is a small part of the $N$ code-word after interleaving. Thus, this scheme provides privacy to the source (user). However, more bandwidth is required compared to the non-cooperation system due to the additional encoding of the second partition $N 2$ as well as some form of repetition of the second partition $N 2$ is included.

$$
B W c c=((3 / 4) /(3 / 5))=1.25 B W n c
$$

Coded cooperation system needs to increase the data rate by a factor of 1.25 to tolerate any message delay especially in real time applications. A tradeoff between diversity and BW must be achieved by our model for optimal performance.
This design with $(35$ symbols $\times 8$ bits $/$ symbol $=280$ bits $)$ input message can support several cooperation levels $(L=N 2 / N)$. The maximum number of bits for cooperation should be chosen so that the number of bits in the output of RSCC2 encoder at the source is less than the number of bits transmitted into the direct path. It can be found for our model, $\mathrm{RS}(N=255, K=239, T=8)$ and convolutional encoder of $1 / 2$ coding rate, by this relation: $N 2$ bits $=(0.25 \times N$ bits -8 bits $=88$ bits $)$, approximately one quarter of the code-word. As shown in Fig. (7), 88 bits cooperation produces 256 bits (less than 296 bits transmitted into the direct channel) in the output of RSCC2. One third of the code-word (120 bits) will produce 320 bits (more than 264 bits transmitted into the direct path). In order that, the first cooperation level is $(88 / 384)$ and the next is $(80 / 384)$ where $(L i-L(i+1)$ 


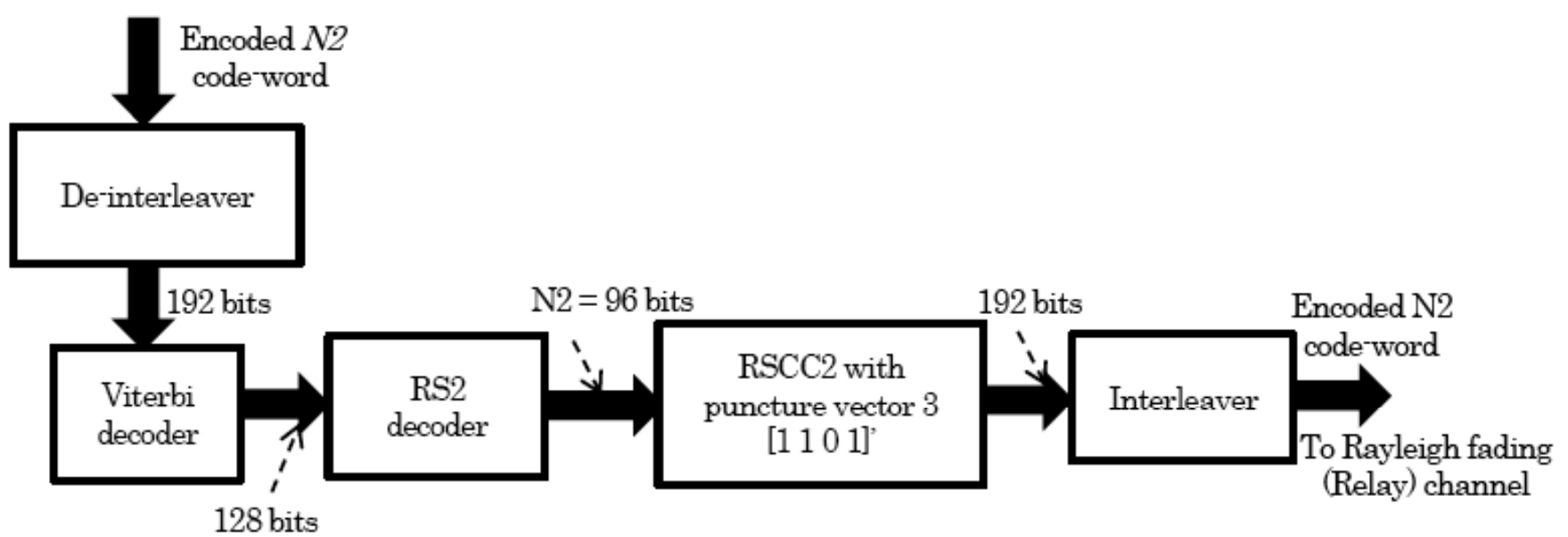

Fig. (8). Coded cooperation at Relay, (88/384) cooperation level, code rate $R \approx 3 / 5$ (model 2).
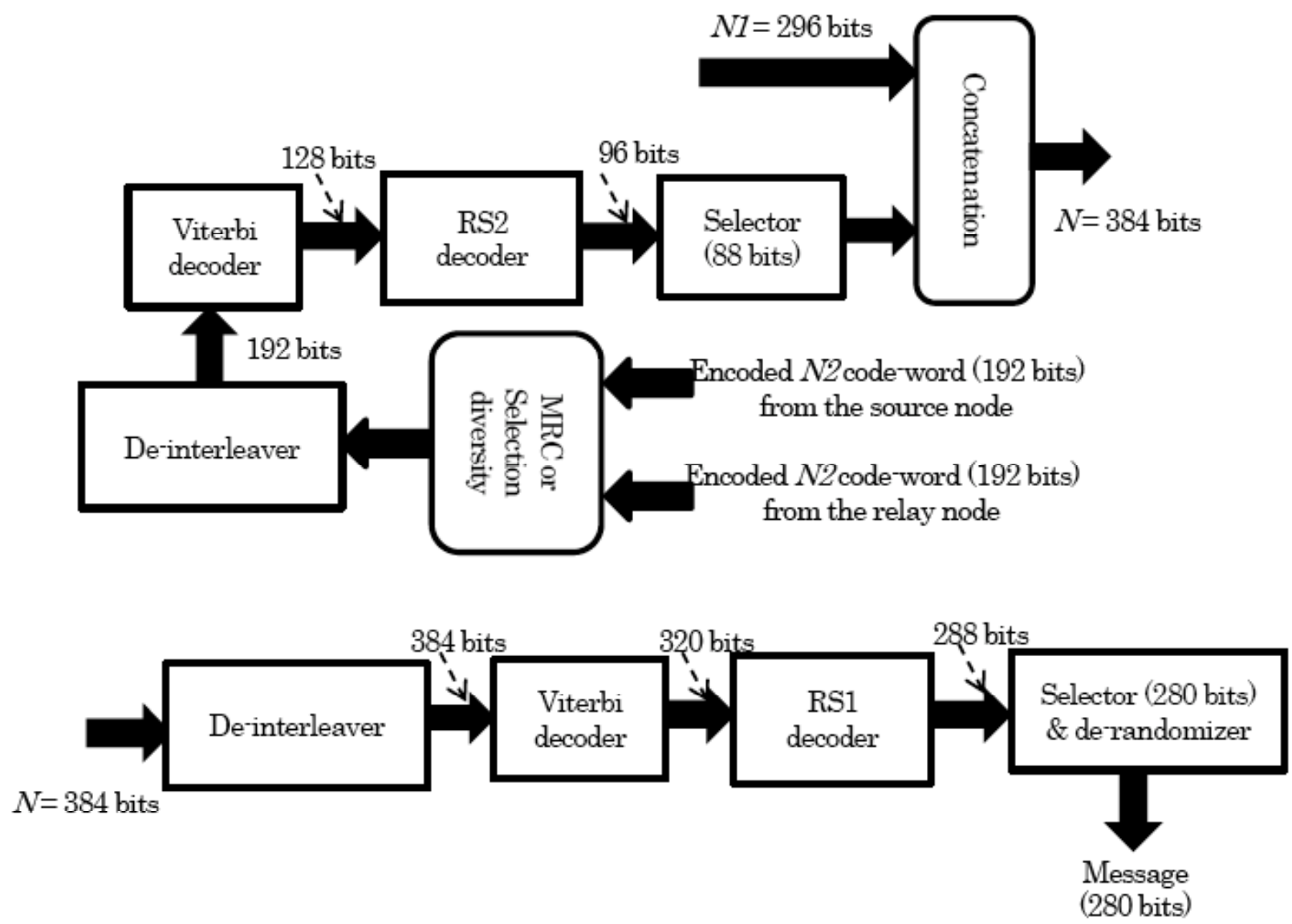

Fig. (9). Coded cooperation at the base station, (88/384) cooperation level, code rate $R \approx 3 / 5$ (model 2).

$=1$ byte). " $i "$ is the order of the cooperation level. The system can adapt the cooperation level depending on the condition of the inter-user channel SNR. Channel state information (CSI) is assumed to be known at each node. When the interuser channel SNR becomes in a bad condition as the relay cannot decode the $N 2$ code-word whatever the system reduces the cooperation level, the system directly reverts to non-cooperative mode.

\subsection{Relay}

As shown in Fig. (8), the relay only decodes the second partition, encoded $N 2$ code-word, to recover $N 2$ segment by
Viterbi and RS decoders. Then N2 segment is encoded again by Reed Solomon and convolutional encoders (RSCC2) if the decoding is successful. After that, N2 is interleaved and transmitted to the base station through Rayleigh fading (relay) channel.

\subsection{Base Station}

At the base station as shown in Fig. (9), encoded N2 segments are combined by (MRC) or simplicity selection diversity can be used for space diversity. Then the output is de-interleaved and decoded to get back N2 segment. Then $N 2$ is concatenated with $N 1$ segment that reached the base 


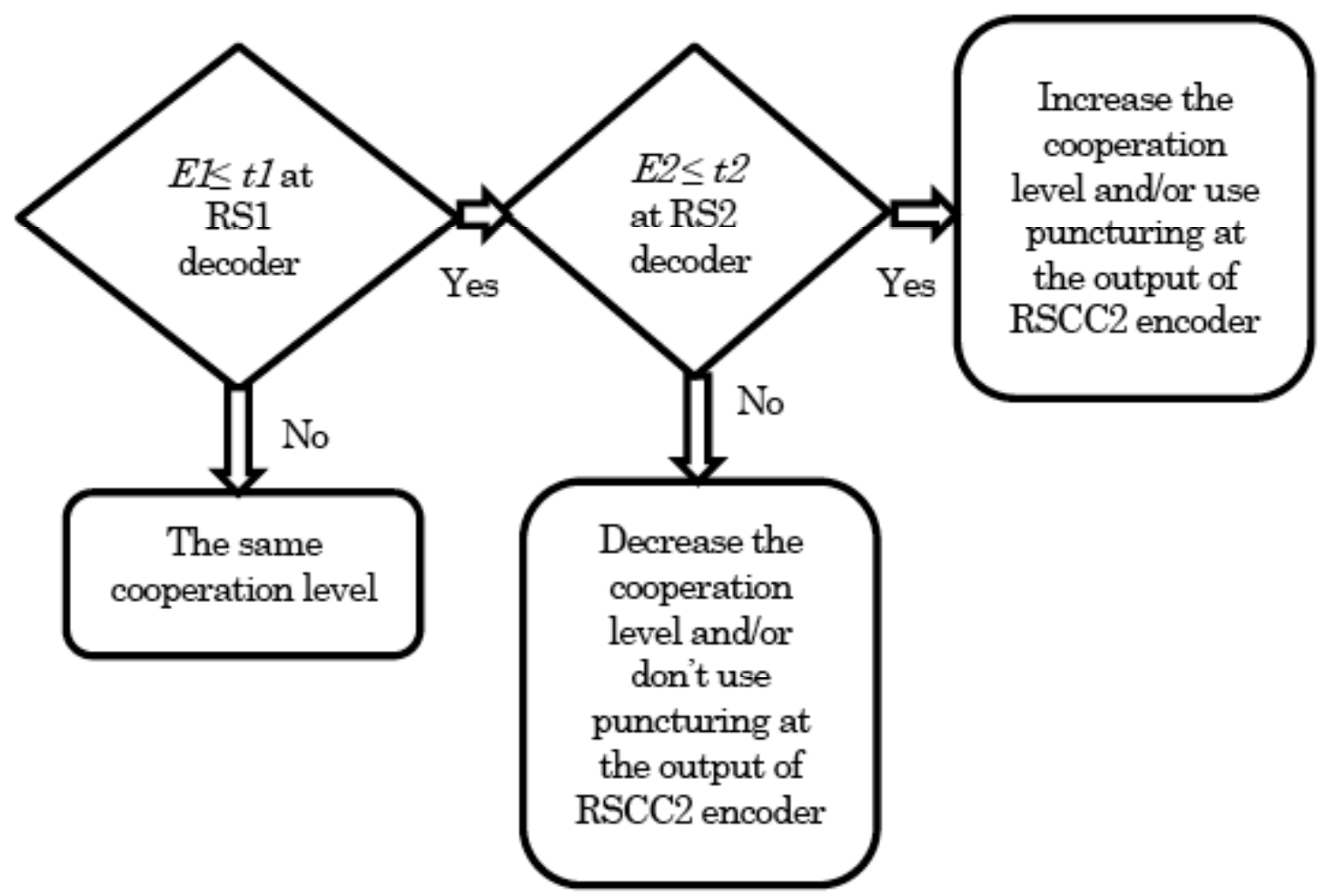

Fig. (10). Adaptive operation of cooperative system (model 2).

station via the direct channel to regain $N$ code-word. After that, $N$ code-word is decoded to recover the original message of the source. RS decoders at the relay and the base station can be used to determine the optimal cooperation level that should be adapted by the system automatically during communication. In this system, RS decoders can correct up to 2 bytes (16 bits) received in errors. It can be computed by the following equation:

$t 1=[(320-288) / 2]=t 2=[128-96) / 2]=16$ bits

Fig. (10) shows the operation of this cooperative system, model 2. This scheme can be described below.

- The system checks RS decoder, RS1, at the base station. If $(E 1<=t 1)$, the system checks if RS2 at the relay decodes the encoded N2 segment successfully.

- If $(E 2<=t 2)$, it means the inter-user channel is good. So the system increases the cooperation level and/or uses puncturing at the output of RSCC2 encoder.

- If $(E 2>t 2)$, it means the inter-user channel is not good. So the system decreases the cooperation level and/or doesn't use puncturing at the output of RSCC2 encoder.

- If $(E 1>t 1)$, it means the direct channel is not good. So the system maintains the current cooperation level without regard to the condition of the inter-user channel.

\section{RESULTS \& DISCUSSION}

The simulation results shown in figures are obtained from both cooperative designs that are considered as follows:

- Two partners (source, relay) are proposed.
- The partner (relay node) is predetermined.

- Reed Solomon, RS (255, 239, T=8), and convolutional, CC $(171,131, L=7)$, codes are utilized. They represent the mandatory forward error correction (FEC) used in IEEE 802.16e WiMAX.

- Channels are subject to the influence of Rayleigh flat fading channels and maximum Doppler shift is assumed to be $100 \mathrm{~Hz}$.

- A single 0X00 byte is appended to each burst for Viterbi decoding algorithm.

- Interleaver and Randomizer employed in IEEE 802.16e WiMAX are adopted here.

- Puncture vector1 ([ $\left.\left[\begin{array}{llllllllll}1 & 1 & 0 & 1 & 1 & 0 & 0 & 1 & 1 & 0\end{array}\right]\right)$ is used for puncturing at the source in both models to puncture the $\mathrm{N}$ code-word.

- Puncture vector2 ([ $\left.\left[\begin{array}{llllllllll}0 & 0 & 1 & 0 & 0 & 1 & 1 & 0 & 0 & 1\end{array}\right]\right)$ is used at the relay in the first model to produce the second partition N2.

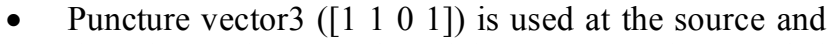
the relay in the second model to puncture the encoded N2 segment.

- Selection diversity is assumed to be the space diversity technique at the base station.

- Both designs are modeled and simulated by MATLAB simulator to get the results.

\subsection{The First Model}

In this model, we compare the cooperative design to noncooperative system in term of bit error rate with varies SNR. Non-cooperative system, with puncturing and without punc- 


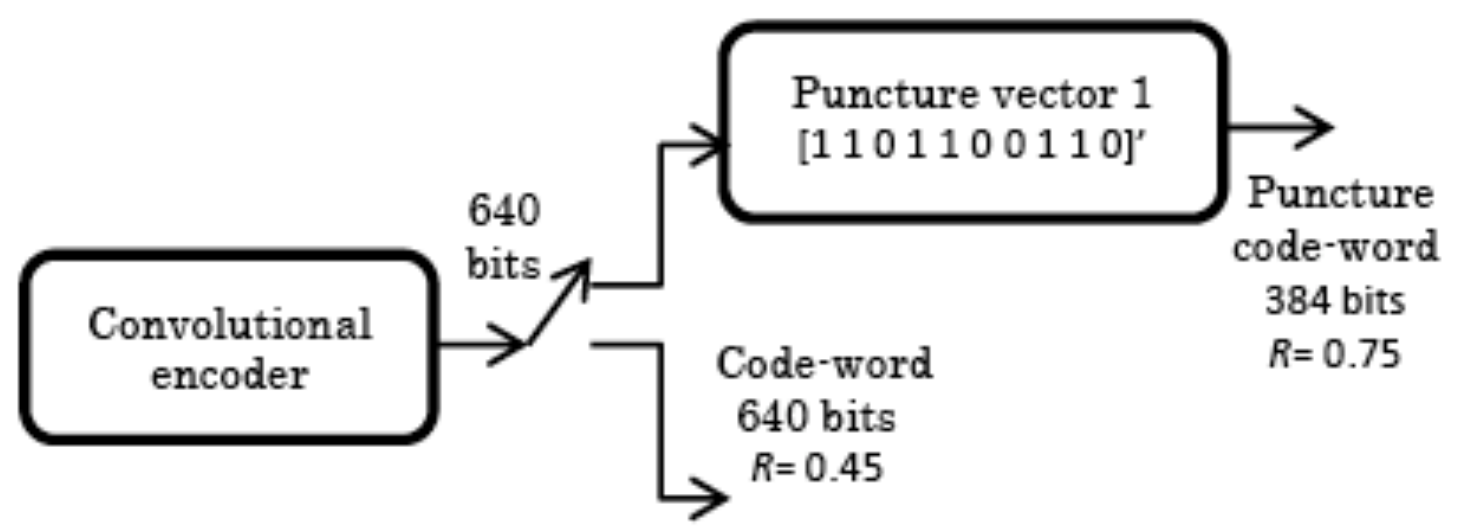

Fig. (11). Non-cooperative system with and without puncturing.

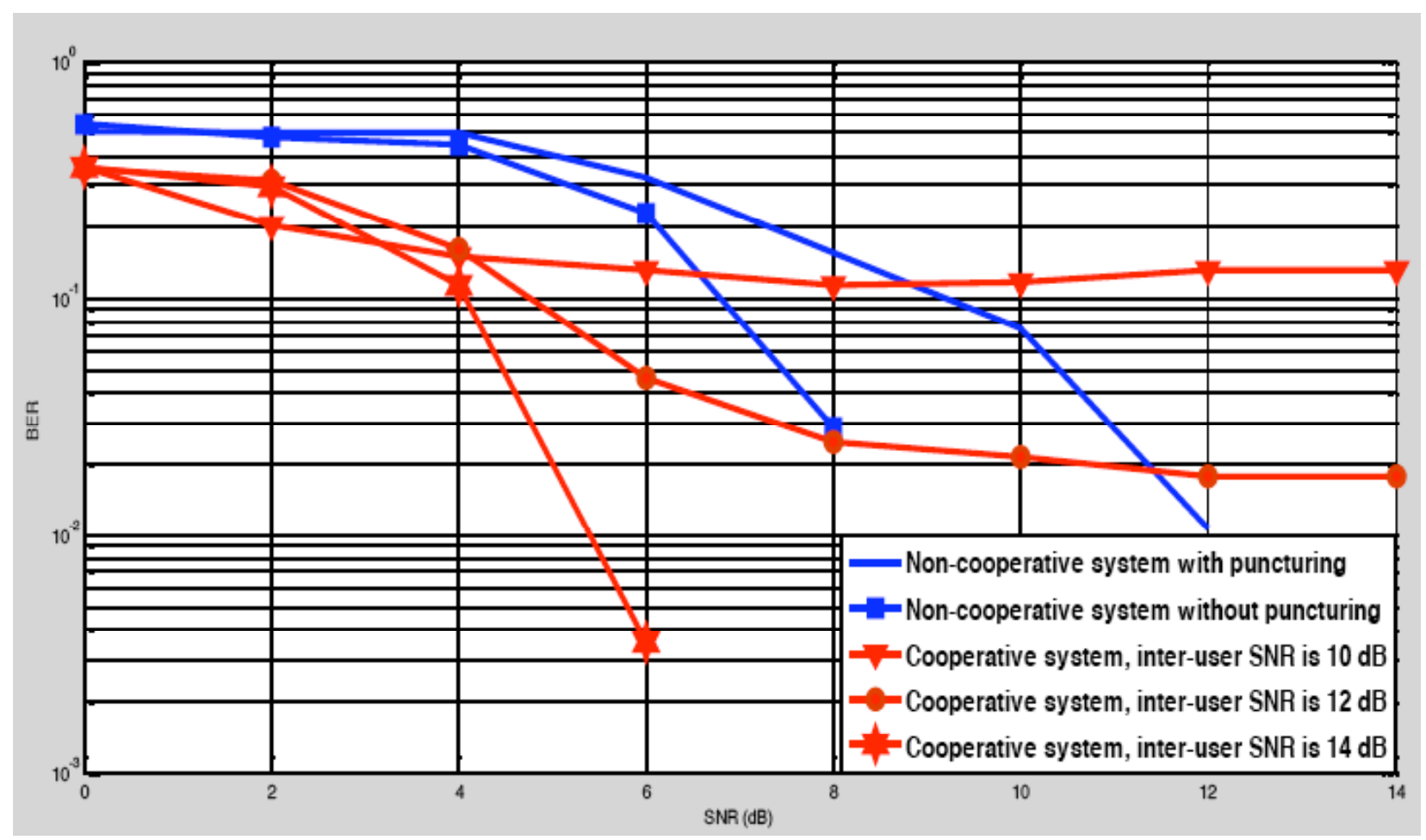

Fig. (12). Comparison of cooperative system with non-cooperative system (with puncturing and without puncturing). Direct and relay channels are identical and vary from zero to $14 \mathrm{~dB}$. Inter-user channel SNR varies $(10,12,14 \mathrm{~dB})$, QPSK for modulation.

turing, is employed to compare its performance to the cooperative system. With puncturing, Non-cooperative system

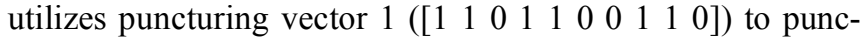
ture code-word from the output of convolutional encoder, whereas without puncturing, non-cooperative system allows code-word to pass from the output of convolutional encoder without puncturing as shown in Fig. (11).

Fig. (12). Illustrates the comparison between the cooperative system to non-cooperative systems with varies inter-user channel SNR.

As shown in Fig. (12), when inter-user channel is good, relay successfully decodes the source's data, the performance of the cooperative scheme is better than noncooperative system with either puncturing or without puncturing. When SNR of the inter-user channel is $14 \mathrm{~dB}$ and BER is $\left(2.86 \times 10^{-2}\right)$, around $3 \mathrm{~dB}$ diversity gain is achieved for the cooperative system over non-cooperative system without puncturing and around $6 \mathrm{~dB}$ over non-cooperative system with puncturing. When the inter-user channel SNR is not good $(<14 \mathrm{~dB})$, relay unsuccessfully decodes the source's data, the performance of the cooperative framework becomes worse than non-cooperative system at high signal to noise ratio (SNR) of the uplink channels.

Actually, the system prefers to work in cooperative mode when relay successfully decodes the source's data. That takes place when the inter-user channel SNR is $\geq 14 \mathrm{~dB}$ (as shown in Fig. (12)) if puncture vector 1 ( $\left(\begin{array}{lllllllll}1 & 1 & 0 & 1 & 1 & 0 & 0 & 1 & 1\end{array}\right.$ $0]^{\prime}$ ') is used as we applied in the first model simulation above since this vector is utilized in IEEE 802.16e WiMAX standard. Although this SNR value of the inter-user channel is high, the cooperative system can decrease the puncturing rate (reduce the length of $N 2$ segment) if the quality of the interuser channel is not good enough to enable relay to successfully decode the source's data. 


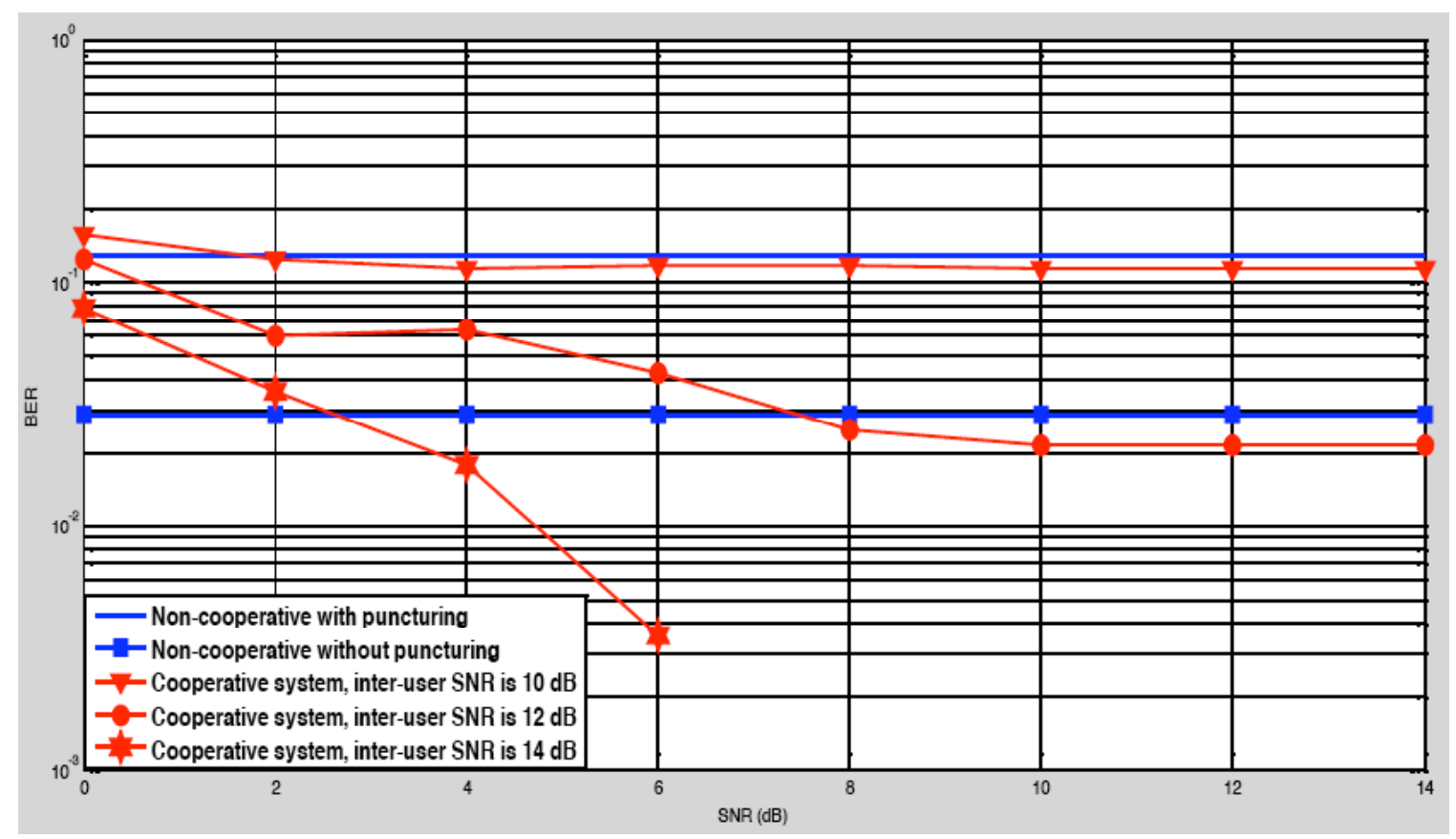

Fig. (13). The performance of cooperative system. Direct channel is fixed to $8 \mathrm{~dB}$ and relay channel varies from zero to $14 \mathrm{~dB}$. Inter-user channel SNR varies with values $(10,12,14 \mathrm{~dB})$, QPSK for modulation.

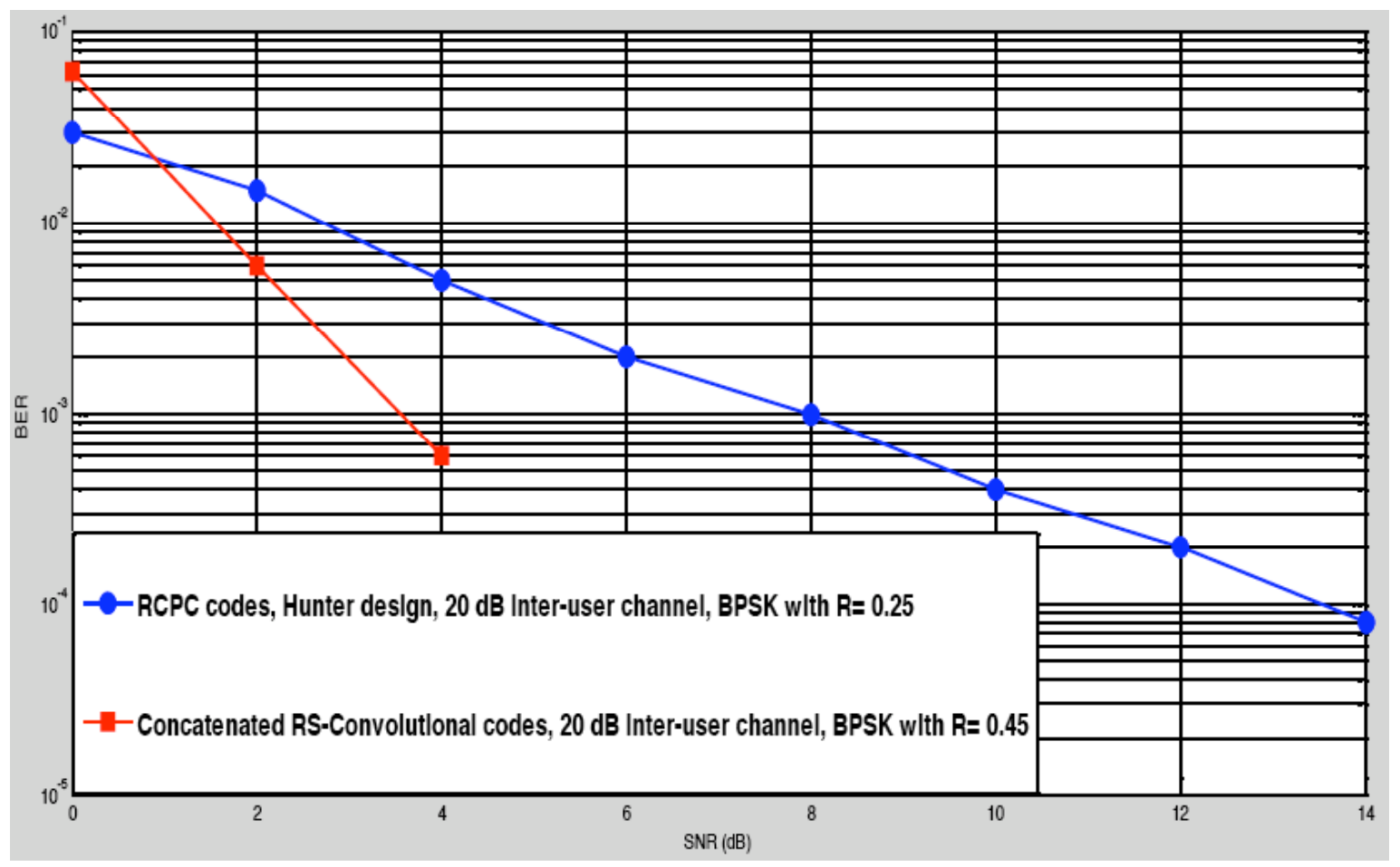

Fig. (14). Comparison of Concatenated RS- Convolutional (RSCC) coded cooperation with RCPC coded cooperation under slow Rayleigh fading, BPSK for modulation.

In Fig. (13), the performance of the cooperative system with varies inter-user channel SNR is addressed when the direct channel SNR is fixed at $8 \mathrm{~dB}$ and relay channel SNR varies from 0 to $14 \mathrm{~dB}$. In $14 \mathrm{~dB}$ inter-user channel SNR, the performance of the cooperative system starts to be better than non-cooperative method without puncturing after around 2.7 $\mathrm{dB}$ relay channel SNR while this cooperative technique exceeds non-cooperative scheme with puncturing in performance at any SNR value of the relay channel when inter-user channel
SNR is higher than $12 \mathrm{~dB}$. At $10 \mathrm{~dB}$ inter-user channel SNR, no diversity gain is achieved due to cooperation. So the system prefers to revert to non-cooperative mode.

To compare the performance of the proposed model to literature review, Fig. (14) illustrates the comparison between Concatenated RS-Convolutional (RSCC) coded cooperation, the proposed design, and Rate-Compatible Punctured Convolutional (RCPC) coded cooperation, Hunter 


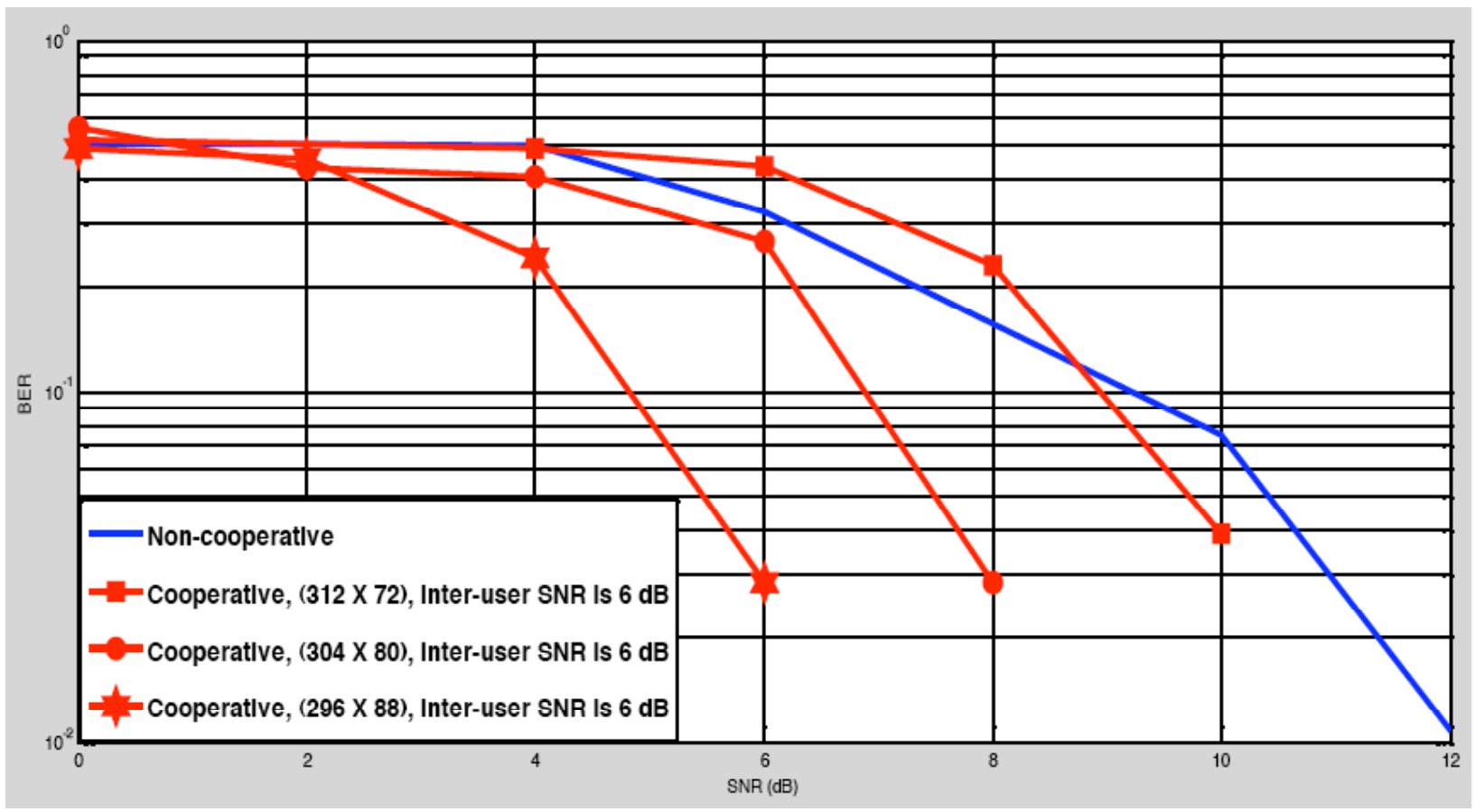

Fig. (15). The performance of cooperative system with several cooperation levels. Inter-user channel SNR is 6 dB. Uplink channels are identical and vary from zero to $14 \mathrm{~dB}$, QPSK for modulation.

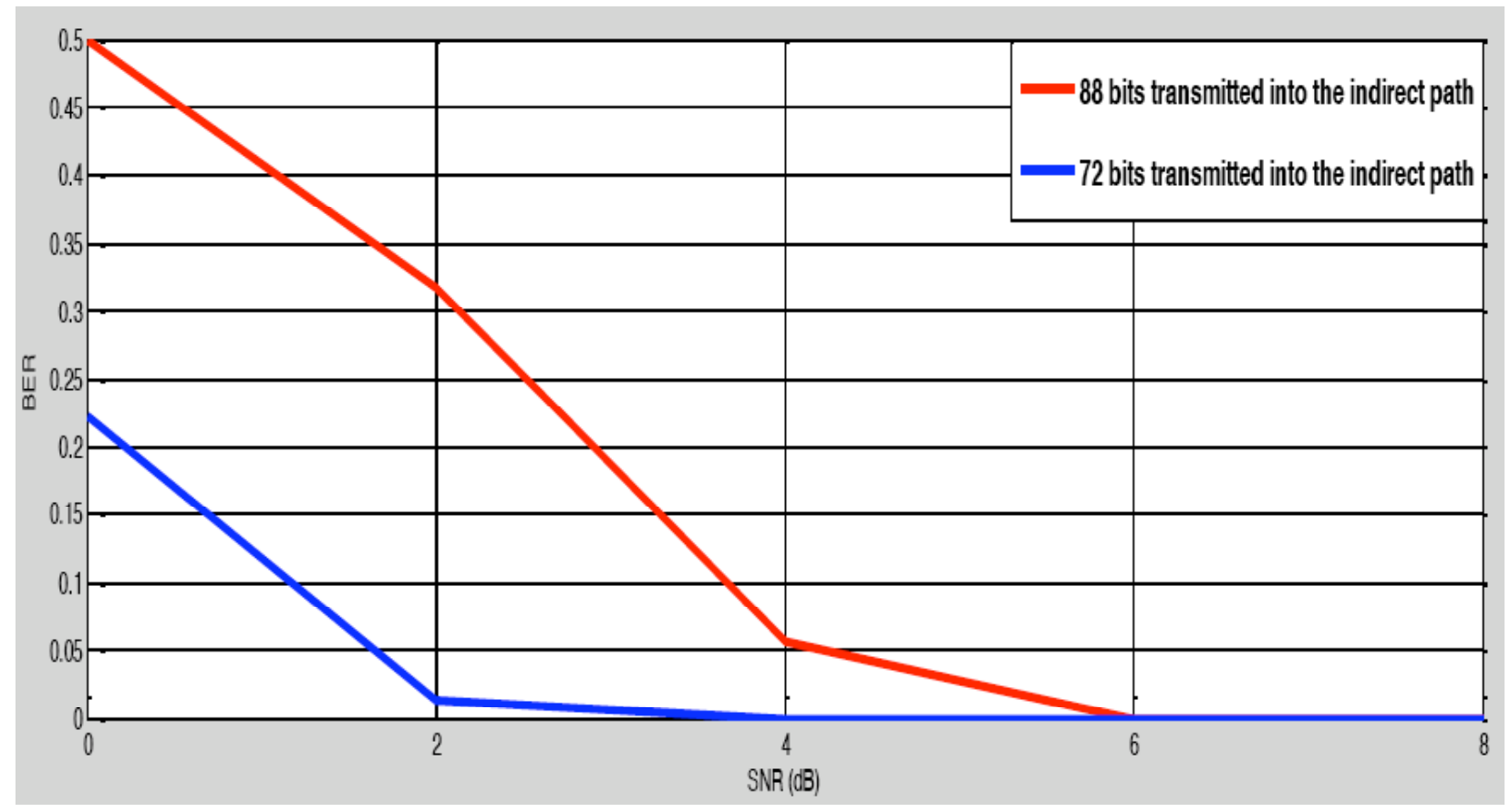

Fig. (16). The BER as a function of SNR of the indirect path, source to relay to base station, inter-user and relay channels are similar and change from zero to $8 \mathrm{~dB}$, QPSK for modulation.

design [6], under slow Rayleigh fading channel. Since Hunter and Nosratinia in their design chose BPSK for modulation, we adopt BPSK in the following simulation to make a precise comparison.

Despite the code rate $(R=0.25)$ of RCPC is less than the code rate $(R=0.45)$ of RSCC, the performance of RSCC coded cooperation is much better than RCPC coded cooperation. It means RSCC provides more information rate with better performance. The outperformance of our system indi- cates RSCC, which consists of Reed Solomon and Convolutional codes, is more effective than RCPC, which consists of $\mathrm{CRC}$ and Convolutional codes, in reducing the bit error rate (BER) over Rayleigh fading channels.

\subsection{The Second Model}

In Fig. (15), the performance of coded cooperation communication scheme with different cooperation levels is compared to the system without cooperation when the inter-user 
channel SNR is good and the uplink, direct and relay, channels are similar and changes from $0 \mathrm{~dB}$ to $14 \mathrm{~dB}$. Three cooperation levels- (296 X 88), (304 X 80), and (312 X 72) for the cooperative system are addressed and inter-user channel SNR is set at $6 \mathrm{~dB}$. Since the inter-user channel is good, the performance of (296 X 88) cooperative scheme is better than others. More bits are encouraged to take the indirect path from source to relay and then to base station.

So the source chooses $(88 / 384)$ cooperation level for cooperative mode to achieve the highest diversity gain. When BER is $\left(2.86 \times 10^{-2}\right)$, around $5 \mathrm{~dB}$ diversity gain is obtained due to (296 X 88) cooperation level and around $3 \mathrm{~dB}$ due to (304 X 80) cooperation level and around $1 \mathrm{~dB}$ due to $(312 \mathrm{X}$ 72) cooperation level at high signal to noise ratio of the uplink channels.

Fig. (16) shows the BER as a function of the indirect path SNR. As illustrated in Fig. (16), the blue curve, when 72 bits are transmitted into the indirect path, drops to zero (no errors) faster than the red curve, when 88 bits are transmitted. So at low inter-user channel SNR (less than $6 \mathrm{~dB}$ ), the performance of the system with $(312 \times 72)$ cooperation level is better than with $(296$ X 88) cooperation level. As illustrated in Fig. (10), the system decreases the cooperation level when the relay fails to decode the second partition (N2) due to the bad condition of the inter-user channel SNR until the relay successfully decodes the second partition (N2) or source reverts to non-cooperative mode.

\section{CONCLUSION}

This work presents two different coded cooperation schemes applied to the mandatory specifications of IEEE 802.16e WiMAX, Reed Solomon (RS) and convolutional (CC) coders.

After analyzing their performance, it's shown that magnificent diversity gains are achieved over non-cooperative system.

The first design achieves diversity gain over noncooperative system with the same information rate $(R=0.45)$ and bandwidth. Also the first model supports more information rate with better performance than RCPC coded coopera- tion system adopted by Hunter and Nosratinia [6] under slow Rayleigh fading channel.

The second design provides privacy for the source, user, as the partition is executed after interleaving the code-word as well as the relay, partner, only receives a small part, around one quarter, of the total code-word as a maximum. The second design also provides a method for the system to adapt its cooperation level according to the channels conditions. Nevertheless, this model supports diversity gain over non-cooperative system with more required bandwidth as well as some form of repetition of the second partition N2 is included.

\section{CONFLICT OF INTEREST}

The authors confirm that this article content has no conflicts of interest.

\section{ACKNOWLEDGEMENT}

Declared none.

\section{REFERENCES}

[1] J. N. Laneman, G. W. Wornell, and D. N. C. Tse, "An efficient protocol for realizing cooperative diversity in wireless networks,", Proceedings IEEE International Symposium on Information Theory, 2001, p. 294.

[2] A. Sendonaris, E. Erkip, and B. Aazhang, "User cooperation diversity. Part I. System description," IEEE Trans. Commun., vol. 51, pp. 1927-1938, 2003.

[3] A. Sendonaris, E. Erkip, and B. Aazhang, "User cooperation diversity. Part II. Implementation aspects and performance analysis," IEEE Trans. Commun., vol. 51, pp. 1939-1948, 2003.

[4] A. Nosratinia, T. E. Hunter, and A. Hedayat, "Cooperative communication in wireless networks," Commun. Mag. IEEE, vol. 42, pp. 74-80, 2004

[5] T. E. Hunter and A. Nosratinia, "Coded cooperation under slow fading, fast fading, and power control," In Conference Record of the Thirty-Sixth Asilomar Conference on Signals, Systems and Computers, Texas University, TX, USA 2002, vol. 1, pp. 118-122.

[6] T. E. Hunter and A. Nosratinia, "Diversity through coded cooperation," IEEE Trans. Wireless Commun., vol. 5, pp. 283-289, 2006.

[7] T. E. Hunter, S. Sanayei, and A. Nosratinia, "Outage analysis of coded cooperation," IEEE Trans. Inform., Theor., vol. 52, pp. 375391, 2006.

[8] G. B. S. R. Naidu. Telagarapu, K.Chiranjeevi, "Analysis of Coding Techniques in WiMAX," Int. J. Comput. Appl., vol. 22, p. 8, 2011.

[9] T. S. Rappaport, "Practical Space Diversity Considerations," In Wireless Communications Principle and Practice, M. Vincenti, Bernard M. Goodwin, Eds., $2^{\text {nd }}$ ed: 2002, pp. 385-387.

(C) Al-Moliki et al.; Licensee Bentham Open.

This is an open access article licensed under the terms of the Creative Commons Attribution Non-Commercial License (http://creativecommons.org/licenses/by-nc/3.0/) which permits unrestricted, non-commercial use, distribution and reproduction in any medium, provided the work is properly cited. 